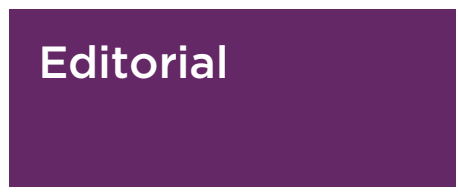

\title{
Journal of Molecular Endocrinology 25th anniversary special issue
}

\section{Ronald M Evans}

Investigator, Howard Hughes Medical Institute and Professor, Salk Institute for Biological Studies, La Jolla, CA, USA

Correspondence should be addressed

to R M Evans

Email

evans@salk.edu or

cbrondos@salk.edu
Nuclear receptors (NRs) comprise a large family of signal dependent transcription factors that respond to a diverse range of fat soluble ligands including steroids, vitamins A and $\mathrm{D}$, long chain fatty acids, bile acids, thyroid hormone and xenobiotic toxins. Through their cognate receptors, nuclear-active hormones orchestrate a multiplicity of biological functions from embryonic development to endocrine physiology, pathology, and therapeutics. Since the cloning of the human glucocorticoid receptor (hGR) in 1985 and the estrogen receptor (ER) in 1986 this family has grown to include 48 unique receptors integrally linked to the evolution of the entire animal kingdom. Intense research efforts have been dedicated to understanding the molecular mechanism of nuclear hormone signaling and how receptor interactions with the genome influence basic biology. At a structural level, NR-mediated transcriptional activation is a complex, multistep process orchestrated primarily by the concerted actions of a DNA binding domain (DBD) and a ligand binding domain (LBD) incorporating coregulator binding sites. While initial structural studies on isolated domains provided a fundamental understanding of the discrete functions of these domains, a fully integrated picture on how NRs are dynamically regulated to enact a coordinated gene transcription response was lacking. Newly emerging crystal structures, reviewed by Fraydoon Rastinejad, are now shedding light on the quaternary arrangement of NR protein complexes, with direct implications for drug discovery and design (Rastinejad et al. 2013). Concurrently, tremendous advances in structural studies of coregulator proteins, reviewed by John Schwabe, are revealing how NRs orchestrate protein complexes that modify chromatin to activate or repress gene transcription (Millard et al. 2013).
These reviews detail the major advances in our understanding of the molecular mechanisms of nuclear receptor action achieved through structural studies. However, for many decades, studies of NR action at the chromatin level were limited to promoters of wellcharacterized target genes. Perhaps one of the foremost questions of nuclear receptor biology has been how a single hormone responsive transcription factor, reading the same DNA template, is capable of regulating different genes in different cell types. Recent advances in high content DNA sequencing technology have revolutionized the study of NR signaling, allowing interrogation of DNA bound sites on a genome-wide scale. Focusing on the macrophage, Christopher Glass explains how whole genome sequencing has revealed how modifications of the epigenetic landscape driven by lineage determining factors dictate NR binding sites and thereby target genes (Zhang \& Glass 2013). Furthermore, untargeted sequencing approaches have revealed that the majority of NR binding sites are located not in promoter regions but in inter- and intra-genic locations that exhibit features of enhancers. Many of these enhancer sites appear to be functional, as evidenced by the production of enhancertemplated non-coding RNA (eRNA). This area is rapidly evolving and fundamentally changing our view of how NRs signal at the chromatin level.

Consistent with the importance of NR signaling on whole body metabolic homeostasis, endogenous ligand production and degradation must be tightly regulated. However, in the setting of metabolic disease such as obesity, disruption of NR ligand levels can lead to dysregulated NR signaling that contributes to and augments the severity of the disease. Evan Simpson reviews how chronic inflammation associated with high fat diet

Published by Bioscientifica Ltd.

This editorial accompanies the 25th anniversary special issue of Journal of Molecular

Endocrinology. The Guest Editor for this section was Ropald M. Evans, Investigator, $08: 55: 16 \mathrm{AM}$

Howard Hughes Medical Institute and Professor, Salk Institute for Biological Studies, La Jolla, CA, USA 
disrupts the normally tight regulation of the aromatase gene. Aromatase is involved in the biosynthesis of estrogen, the ligand for the estrogen receptor (ER). Chronic inflammation, driven by cytokines and inflammatory signaling molecules, induces aromatase gene expression that increases estrogen production. Increased estrogen signals through its cognate receptor to increase cell proliferation and the risk of breast cancer in postmenopausal women (Simpson \& Brown 2013).

Two ER receptors are expressed in humans, ER $\alpha$ and $\mathrm{ER} \beta$, both of which are involved in the etiology and/or the progression of different cancers including prostate, breast and ovarian cancer. Jan-Ake Gustafsson reviews for us the complex biology and mechanism of ER action throughout the body and focuses on the divergent roles of these two closely related receptors (Dey et al. 2013). ER $\alpha$ is an established oncogene while ER $\beta$ appears to function as a tumor suppressor. This recent distinction has focused drug discovery efforts towards identifying $\mathrm{ER} \alpha$ antagonists and ER $\beta$ selective agonists; novel ER $\beta$ agonists are currently being evaluated for cancer prevention as well as other diseases.

The position of NRs between cellular and whole body systems and the genome, combined with their liganddependent activity, has identified them as therapeutically accessible targets for metabolic disease, particularly relevant in the emerging era of pandemic obesity. While adipose tissue was an obvious player in obesity, the discovery of adipokines and their regulatory roles in metabolism, energy intake, and fat storage has promoted studies in this tissue. White adipose tissue (WAT) has been intensively studied while studies in brown adipose tissue (BAT) were restricted by its perceived absence in humans. However, the recent confirmation of BAT in humans has stimulated an intense focus on BAT biology and may offer unique opportunities to treat obesity and its metabolic consequences. Antonio Vidal-Puig reviews the latest insights and controversy surrounding BAT development and activation in rodents and humans, focusing on similarities and functional differences between BAT and the newly discovered brown-in-white (brite) adipose cells (Carobbio et al. 2013).

In an ideal world, dietary restriction and exercise can overcome obesity. Exercise activates the AMP-activated protein kinase (AMPK) in muscle and other tissues, a pathway that increases fat oxidation and glucose transport. These changes are driven by a network of incompletely understood molecular pathways that trigger transcriptional remodeling of the skeletal muscle. Ronald Evans reviews recent advances in the understanding of key NR signaling components of this circuitry including peroxisome proliferator activator receptors (PPARs), estrogen-related receptor (ERRs), Rev-erbs, their associated co-regulators and their interactions with AMPK, that govern aerobic transformation of the skeletal muscles (Fan et al. 2013). Johan Auwerx reviews the role of acetylation in this pathway and its essential role in intermediary muscle metabolism (Menzies \& Auwerx 2013). Acetylation is a dynamic protein modification and serves as an essential rheostat in muscle. Dysregulated acetylation associated with metabolic disease results in aberrant acetylation of key proteins that control cellular substrate levels as well as mitochondrial content and function. Both of these reviews highlight recent discoveries that raise the possibility of synthetically mimicking exercise with pathway-specific drugs to improve aerobic capacity and, in turn, health.

Finally, a comprehensive review from Mitchell Lazar on orphan receptors, so named because of their apparent lack of endogenous ligands, completes this special issue (Mullican et al. 2013). NR orphan receptors were originally identified by sequence homology to pioneer members of the family like glucocorticoid, estrogen and the thyroid hormone receptors. Lazar reviews the current successes and problems with identifying cognate endogenous ligands for this class of receptor, as well as with the identification of synthetic ligands and genetic models that have helped us understand and elucidate the biological targets and roles of these receptors. The importance of these receptors as pharmacological targets in the foreseeable future is readily apparent.

In conclusion, this series of reviews, presented in this 25th anniversary special issue of Journal of Molecular Endocrinology, demonstrates how far the field of endocrinology has come in understanding how the family of nuclear-active lipophilic hormones regulates body metabolism at the genomic level, as well as identifying novel pathways and candidate targets that can be pharmacologically manipulated to improve human health from controlling metabolic disease to combating cancer.

\section{References}

Carobbio S, Rosen B \& Vidal-Puig A 2013 Adipogenesis: new insights into brown adipose tissue differentiation. Journal of Molecular Endocrinology 51 T75-T85. (doi:10.1530/JME-13-0158)

Dey P, Barros RPA, Warner M, Ström A \& Gustafsson J-Å 2013 Insight into the mechanisms of action of estrogen receptor $\beta$ in the breast, prostate, colon, and CNS. Journal of Molecular Endocrinology 51 T61-T74. (doi:10. 1530/JME-13-0150)

Fan W, Atkins AR, Yu RT, Downes M \& Evans RM 2013 Road to exercise mimetics: targeting nuclear receptors in skeletal muscle. Journal of Molecular Endocrinology 51 T87-T100. (doi:10.1530/JME-13-0258) 
Menzies K \& Auwerx J 2013 An acetylation rheostat for the control of muscle energy homeostasis. Journal of Molecular Endocrinology $\mathbf{5 1}$ T101-T113. (doi:10.1530/JME-13-0140)

Millard CJ, Watson PJ, Fairall L \& Schwabe JWR 2013 An evolving understanding of nuclear receptor coregulator proteins. Journal of Molecular Endocrinology 51 T23-T36. (doi:10.1530/JME-13-0227)

Mullican SE, DiSpirito JR \& Lazar MA 2013 The orphan nuclear receptors at their 25-year reunion. Journal of Molecular Endocrinology 51 T115-T140. (doi:10.1530/JME-13-0212)
Rastinejad F, Huang P, Chandra V \& Khorasanizadeh S 2013 Understanding nuclear receptor form and function using structural biology. Journal of Molecular Endocrinology 51 T1-T21. (doi:10.1530/JME-13-0173)

Simpson ER \& Brown KA 2013 Obesity and breast cancer: role of inflammation and aromatase. Journal of Molecular Endocrinology 51 T51-T59. (doi:10.1530/JME-13-0217)

Zhang DX \& Glass CK 2013 Towards an understanding of cell-specific functions of signal-dependent transcription factors. Journal of Molecular Endocrinology 51 T37-T50. (doi:10.1530/JME-13-0216)
Published by Bioscientifica Ltd 